\title{
A Study on Idiosyncratic Handwriting with Impact on Writer Identification
}

\author{
Chandranath Adak ${ }^{* \dagger}$, Bidyut B. Chaudhuri ${ }^{\ddagger}$, Michael Blumenstein*† \\ ${ }^{*}$ Centre for AI, School of Software, University of Technology Sydney, Australia-2007 \\ ${ }^{\dagger}$ IIIS, School of ICT, Griffith University, Gold Coast, Australia-4222 \\ ${ }^{\ddagger}$ CVPR Unit, Indian Statistical Institute, Kolkata, India-700108 \\ Chandranath.Adak@student.uts.edu.au
}

\begin{abstract}
In this paper, we study handwriting idiosyncrasy in terms of its structural eccentricity. In this study, our approach is to find idiosyncratic handwritten text components and model the idiosyncrasy analysis task as a machine learning problem supervised by human cognition. We employ the Inception network for this purpose. The experiments are performed on two publicly available databases and an in-house database of Bengali offline handwritten samples. On these samples, subjective opinion scores of handwriting idiosyncrasy are collected from handwriting experts. We have analyzed the handwriting idiosyncrasy on this corpus which comprises the perceptive ground-truth opinion. We also investigate the effect of idiosyncratic text on writer identification by using the SqueezeNet. The performance of our system is promising.
\end{abstract}

Index Terms-Handwriting idiosyncrasy, Inception network, SqueezeNet, Writer identification.

\section{INTRODUCTION}

Handwriting is a nexus of graphical marks/symbols where a human writer can be perceived as a "stochastic pattern generator" of such symbols [1]. The structural pattern of such mark/symbol varies with individual due to personal writing style. On an individual handwritten sample, it can be noted that some marks/symbols are written in an unconventional way and referred to as "handwriting idiosyncrasy" 1 According to the Oxford dictionary ${ }^{2}$ the word "idiosyncrasy" has been originated from the Greek "idiosunkrasia", i.e. idios (own, private) $+\operatorname{sun}$ (with) + krasis (mixture), which suggests "a distinctive or peculiar feature or characteristic" of an individual.

In this paper, the idiosyncratic handwriting interprets individual eccentricities in penning a character. In Fig. 1.(a), a typical idiosyncratic style of writing English character ' $\mathrm{t}$ ' is shown within a red box. Here, the horizontal stroke of ' $t$ ' is scribbled with a continuity of the vertical stroke. Hence, this ' $t$ ' apparently looks like cursive lowercase ' $f$ ' (' $f$ ') and depicts individual idiosyncrasy. Also, Fig. 1 (b) presents an example of Bengali idiosyncratic handwriting, where the redboxed character ' $প$ ' is drawn by noodle-like continuous stroke and creating a queer pattern. Although in this paper, we mainly consider a complex Indic script Bengali (endonym, Bangla) [2], [3], an example in English is presented in Fig. 1] (a) for mass readership.

\footnotetext{
${ }^{1}$ http://www.scottishhandwriting.com , The National Records of Scotland.

${ }^{2}$ https://en.oxforddictionaries.com , retrieved 22 June 2018.
}

(a)

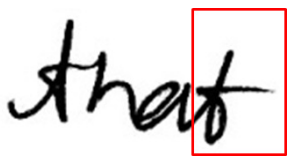

(b)

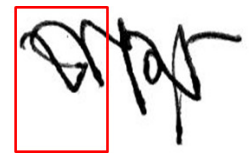

Fig. 1. Examples of highly idiosyncratic handwritten character in (a) English and (b) Bengali script, enclosed in red colored boxes.

In Fig. 2, we present an example of a Bengali text line portion where two idiosyncratic characters (' $\mathrm{k}$ ' and 'ই') occur multiple times. Here, a bigger unusual circular loop (O) instead of the lower dot component of character ' $র$ ' and a black ribbon (' $\ell$ ') like shape in the upper zone of character 'ই' make these highly idiosyncratic.
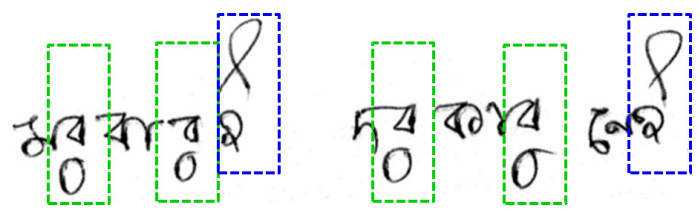

Fig. 2. Two idiosyncratic characters marked by green and blue dotted boxes.

In Fig. 3, we show some examples of idiosyncratic textpatches from the Bengali handwritten old manuscript of Nobel Laureate R.N. Tagore. Fig. 3. (a) depicts the character ' $প$ ' written in a single stroke without lifting the pen, Fig. 3.(b) presents the character ' $\overline{ }$ ' looks more likely Bengali numeral ' character ' $\mathrm{s}$ '. (a)

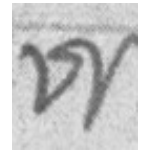

(b)

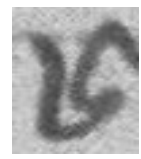

(c)

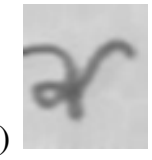

Fig. 3. Highly idiosyncratic text-patches from R.N. Tagore's manuscript.

In the Educational research domain, very few studies have been performed on idiosyncratic writing [4]. On the other hand, in the research field of Document Image Analysis, some works have mentioned handwriting idiosyncrasy with a motivation of data synthesis [5], [6]. We have not found any other references relevant to our work. 
With the assistance of some handwriting experts, we have noted some possible influences of idiosyncratic handwriting generation as follows:

i) Stroke end/start position: The current character shape may depend on the stroke ending position of the previous character and stroke starting positing of the next character.

ii) Positional occurrence: A character shape sometimes becomes eccentric due to its start/middle/end position in a word.

iii) Character stroke component: A single stroke is considered as the scribbling between a pen-down to an immediately next pen-up. Multiple pen-down/ups introduce several character stroke components. Sometimes, to maintain a continuous writing stroke, writer reduces the number of pen-down/ups and may invite idiosyncrasy.

iv) Cursiveness: The cursive Bengali writing influences mostly to the individual writing styles and eccentricities due to lack of proper handwriting teaching/learning curriculum in school [7]. The students learn cursive writing by their own adaptive skills and sometimes by mimicking the handwritings of some family members, teachers, friends, and scholars. Therefore, sometimes family/cultural background becomes influential.

v) Illness: Some illness such as Parkinson's disease, Alzheimer, Dyslexia, Tourette syndrome etc. may be the cause of idiosyncratic handwriting.

This paper is an early attempt of computer-based analysis of idiosyncratic handwriting. Here, we formulate our idiosyncrasy analysis task as a feature-based classification problem and use an auto-derived feature-based Inception network [8]. We also take assistance from several human handwriting experts to provide some cognitive opinion score on handwritten text-patches as ground-truth.

The study of idiosyncratic handwriting can be used for:

i) invoking the artificial intelligence into the machine to understand the idiosyncrasy in handwriting,

ii) sorting the handwritten samples on the basis of idiosyncrasy, which can be utilized in the OCR (Optical Character Recognition) preprocessing stage,

iii) scrutinizing the forensic and biometric aspects of handwriting,

iv) investigating distinct penning styles of some famous writers and scholars for the cultural heritage value,

v) examining early/advanced stage of any physical or neurological disorder which may affect writing styles.

Besides handwriting idiosyncrasy analysis, we further conduct an experiment to see the impact of idiosyncratic handwritten text on writer identification. We note that human handwriting experts emphasize some particular characters/textpatches for unknown writer inspection [9]. Here, we try to adopt this technique to investigate on highly idiosyncratic text-patches. The writer identification task is perceived as a multiclass classification problem [10] and the auto-derived feature-based SqueezeNet [11] is used here to tackle this problem.
The rest of the paper is structured as follows. The proposed method is described in Section II Then, Section III provides the experimental results followed by discussions. Finally, the conclusion is drawn in Section IV.

\section{Proposed Method}

A handwritten document is scanned to obtain a digital image and then it undergoes several preprocessing stages. Very small noisy components are removed by a single pass connected component labeling algorithm [12]. Non-textual components such as drawings/doodles are removed using the technique of [13]. Crossed-out/struck-out text, if present in the document image, is also removed by the method of [14]. The characters are segmented using the water reservoir principlebased scheme of [15]. For our task, instead of normalizing the segmented character, we use a fixed size text-patch. The textpatch is selected empirically as a $116 \times 116$ neighbor window around the center of gravity of a segmented character. For our task, it is not mandatory that a text-patch should always contain a single properly segmented character. We feed these text-patches for idiosyncrasy analysis followed by the study on writer investigation.

\section{A. Idiosyncrasy Analysis}

In this subsection, at first we discuss our perception of idiosyncrasy analysis task as a machine learning problem, then we describe our approach to tackle this problem.

1) Problem formulation: The idiosyncratic variation of handwriting is huge in quantity. The sense of idiosyncrasy in writing patterns can be well-examined by human handwriting experts. Here, we attempt to impart the machine with the experts' cognitive experiences instead of some statistical handcrafted features/characteristics.

The individual text-patches are shown to the human experts to study the idiosyncrasies. The experts are then requested to provide a subjective idiosyncratic score within a continuous range of $\left[I_{1}, I_{2}\right] ; I_{1}, I_{2} \in \mathbb{R}$. The subjective scores are marked in a continuous range to avoid the restriction of scoring on a fixed discrete opinion scale, e.g. Likert scale [16]. For each

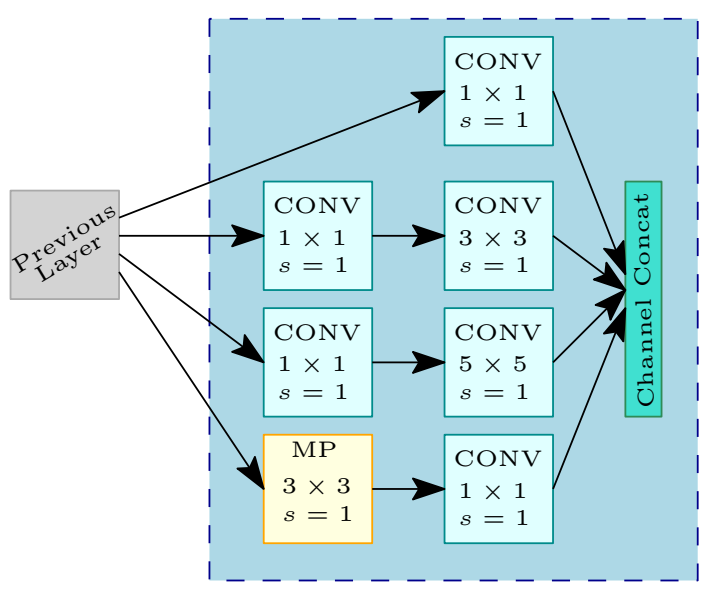

Fig. 4. Inception Module (IM). 


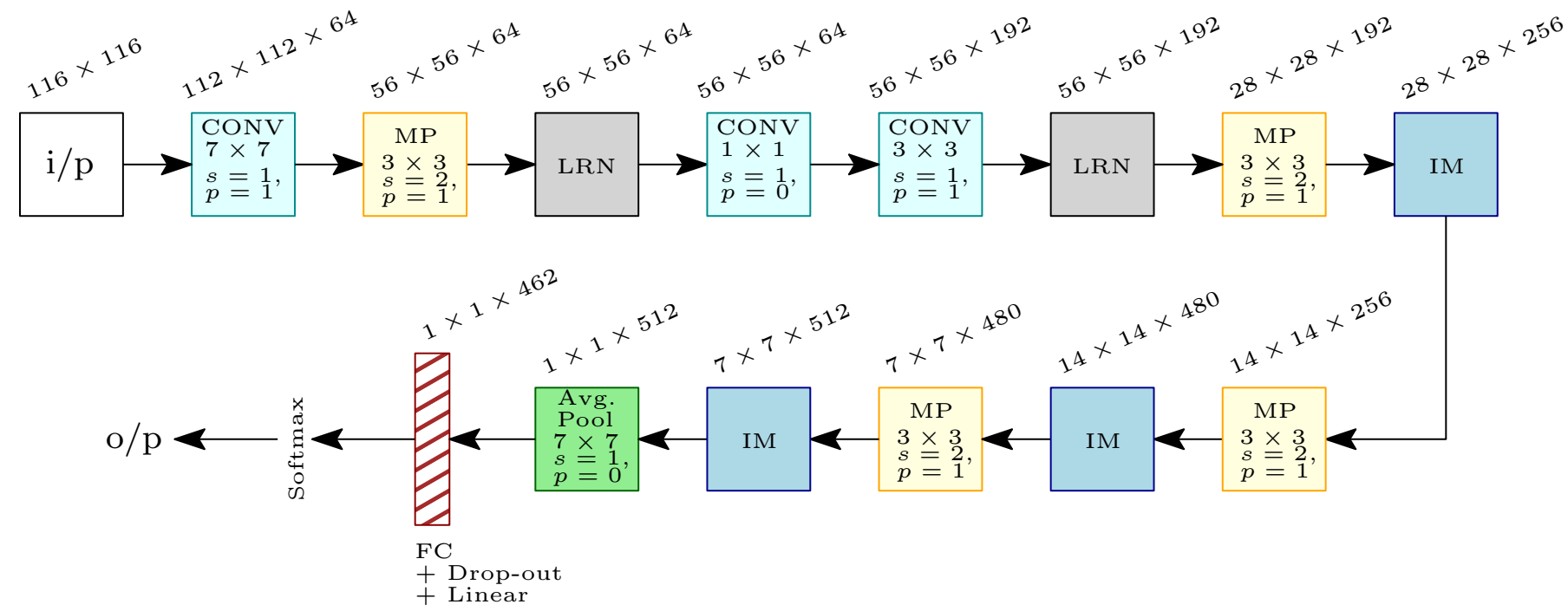

Fig. 5. Inception Network.

text-patch, multiple individuals provide their opinion scores. The arithmetic mean of all these scores is calculated and labeled with each text-patch as its mean opinion score.

After the data collection, the idiosyncratic score range $\left[I_{1}, I_{2}\right]$ is partitioned into $n_{I}$ number of bins of equal span. Therefore, all the idiosyncratic mean opinion scores of entire text-patch set fall into these $n_{I}$ bins. In other words, all the text-patches are categorized into $n_{I}$ classes with respect to the idiosyncratic score.

Thus, we map the idiosyncrasy analysis job into a classification problem. Now, the task is to label a given text-patch into $n_{I}$ classes of idiosyncratic opinion score. Such categorization of opinion scores is a well-known topic in the field of video quality assessment [17].

The idiosyncrasy analysis task is performed by an autoderived feature-based Inception network, discussed in the following subsection.

2) Idiosyncratic text identification: The idiosyncratic textpatches are marked or classified with respect to the idiosyncratic opinion scores. Some auto-derived features are employed for this purpose and discussed in this subsection.

The standard CNN architecture [18] can be deemed as a knot of two parts: front and rear. The front part is the convolutional part regarded as an automated feature extractor, which takes an image input and produces the feature maps. The rear part is typically an MLP (Multi-Layer Perceptron) used as a classifier. The auto-extracted features are fed to the MLP for obtaining the classified output through the stacked fully connected layers.

For our idiosyncratic text analysis, we adopt the deep neural architecture of [8] which has reported the concept of inception module. In Fig. 4 we present the inception module (IM) used in this paper. Here, the "CONV" represents convolutional layer, "Max Pool" denotes max-pooling layer, $s$ symbolizes the stride value. The employed filter size and stride value are provided in Fig. 4 .
We adopt the strategy of inception module and work with a comparatively smaller version of [8] due to lesser class classification and reduced memory complexity. Our adopted version of the Inception Network is shown in Fig. 5. This architecture takes a fixed-sized image input (i/p). We feed the text-patch image of size $116 \times 116$. In the front part of the Inception network, the initial convolutional layer (CONV) contains 64 feature maps of size $112 \times 112$. Each of these feature maps is connected with a $7 \times 7$ neighborhood of the $\mathrm{i} / \mathrm{p}$ image. Here, stride $(s)=1$ and padding $(p)=1$. The following max-pooling (MP) layer, LRN (Local Response Normalization), CONV layers are displayed in Fig. 5 with the corresponding employed filter size, the number of feature maps, map size, stride, padding etc. The actual inner-view of inception module (IM) of Fig. 5 is presented in Fig. 4 In total, here we employ three inception modules. We apply ReLU (Rectified Linear Unit) [19] as an activation function for all the convolutions including those inside the inception module.

After the last inception module, the rear part of the inception network contains an average pooling (Avg. Pool) layer which obeys the technique of [20]. The following layer is fully connected (FC) with a dropout of $40 \%$ [21]. The linear layer with softmax activation is used for predicting the class output $(\mathrm{o} / \mathrm{p})$. The output size of each layer is shown in Fig. 5 .

\section{B. Writer Identification}

Writer identification is a task of allocating a writer-id on a questioned handwritten sample. In other words, writer identification is basically a multiclass classification problem to detect a writer-class among multiple classes [10]. Here also, we use an auto-derived feature-based classification strategy. For writer identification, we employ the SqueezeNet [11] architecture, since this architecture provides good accuracy for general image processing tasks with lesser parameter compared to the AlexNet [22]. 


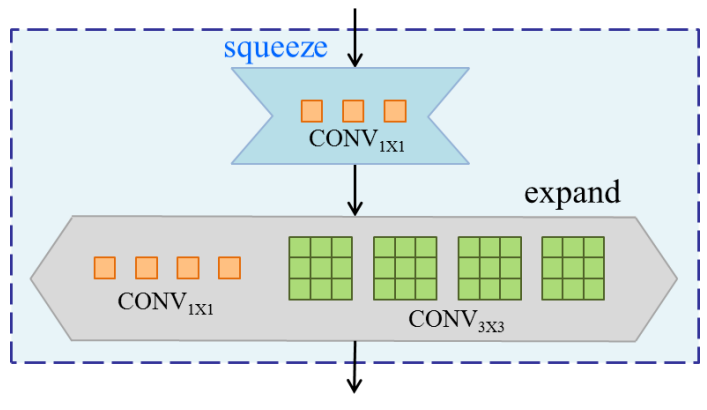

Fig. 6. Fire module.

In SqueezeNet, the key innovation is the fire module (Fire) [11]. A fire module comprises two layers: squeeze and expand, as shown in Fig. 6 The squeeze layer contains $N s_{1 \times 1}$ number of $1 \times 1$ convolutional filters $\left(C O N V_{1 \times 1}\right)$, while the expand layer contains $N e_{1 \times 1}$ number of $1 \times 1$ convolutional filters and $\mathrm{Ne}_{3 \times 3}$ number of $3 \times 3$ convolutional filters $\left(\mathrm{CONV}_{3 \times 3}\right)$. Here, Fig. 6 presents $N s_{1 \times 1}=3, N e_{1 \times 1}=4$, and $N e_{3 \times 3}=$ 4.

For our task, the SqueezeNet takes a text-patch of size $116 \times 116$. The first convolutional layer (CONV) comprises of 96 feature maps of size $112 \times 112$. Every feature map is connected with a $7 \times 7$ neighborhood of the input (i/p) image patch. Here, stride $(s)=1$ and padding $(p)=1$. The following max-pooling (MP), CONV layers are shown in Fig. 7 with the corresponding employed filter size, the number of feature maps, map size, stride and padding measure. Here, we use eight fire modules $\left(\right.$ Fire $_{i \mid i=1,2, \ldots, 8}$ ) in total. The counts of $\left(N s_{1 \times 1}, N e_{1 \times 1}, N e_{3 \times 3}\right)$ in Fire $_{1}$, Fire $_{2}, \ldots$, Fire $_{8}$ are $(16,64,64),(16,64,64),(32,128,128),(32,128,128)$, (48, 192, 192), (48, 192, 192), (64, 256, 256), (64, 256, 256), respectively. The average pooling (Avg. Pool) layer, after the last convolutional layer, follows the technique of [20]. At last, softmax is used to predict the output class $(\mathrm{o} / \mathrm{p})$. We use ReLU (Rectified Linear Unit) [19] as activation function at the end of both squeeze and expand layers of all fire modules. After the eighth fire module $\left(\right.$ Fire $\left._{8}\right)$, a dropout [21] of 50\% ratio is applied.

\section{EXPERIMENTS AND DISCUSSION}

In this section, we discuss our employed database and analyze the efficacy of our system through experiments.

\section{A. Database Employed}

For the experimentation, we required a database having a subjective opinion score of handwriting idiosyncrasy. We did not find any database with proper ground-truth. Therefore, we generated our own corpus containing subjective opinion ground-truth score of idiosyncrasy. For this, we gathered the Bengali writings, on which we requested handwriting experts to put some subjective score while examining the idiosyncrasy, as discussed in Section $\amalg-\mathrm{A} 1$

For the idiosyncratic score, we selected the range $[0,10]$, i.e. $I_{1}=0, I_{2}=10$ (refer to Section II-A1). This range was partitioned into 10 bins, i.e. $n_{I}=10$. Therefore, idiosyncrasy analysis tasks can be perceived as 10-class classification problems. The idea behind such fixing the class number was adopted from [17]. The class- $1\left(\mathrm{ID}_{1}\right)$ was represented by the highest score, i.e. most likely to be an idiosyncratic writing, and class- $10\left(\mathrm{ID}_{10}\right)$ by the lowest score, i.e. least idiosyncratic handwriting.

To obtain the idiosyncratic opinion score, an expert was given cropped Bengali handwritten text-patches irrespective of character class. The expert put the cognitive score by his/her own expertise. For a text-patch, at least 30 human handwriting experts provided the subjective idiosyncratic opinion scores, and the arithmetic mean opinion score was selected as the golden standard for each text-patch.

For subjective opinion score collection, we employed two publicly available databases and one in-house database. The database details are as follows.

i) ICDAR-2013 segmentation [23] (say, $\mathrm{D}_{\mathrm{DAR}}$ ): This database comprises 50 Bengali handwritten pages, made available during ICDAR-2013 handwriting segmentation contest [23].

ii) CMATERdb1.1.1 [24] (say, $\mathrm{D}_{\mathrm{CM}}$ ): This is a subpart of the public database CMATERdb1 [24] and contains 100 Bengali handwritten pages.

iii) In-house database (say, $\mathrm{D}_{\text {new }}$ ): This is an offline database of Bengali handwriting written by 100 writers. Each writer wrote 3 pages to contribute a total of 300 pages.

Here, for $\mathrm{D}_{\text {new }}$ generation, a typical $0.5 \mathrm{~mm}$ ball-point smooth black inked pen of a particular brand/model was used to write on a 75 GSM $\left(g / m^{2}\right)$ blank white page placed on a common good writing surface. The writers were of both genders in the age group of 9 to 67 years, having various academic backgrounds from primary school to university level. The writers were general healthy human-being without having any known serious illness (e.g., Dyslexia, Parkinson's disease etc.) of obstructing general writing. All the handwritings were performed in the healthy awaken state of the writers.

\section{B. Results and Evaluation}

In this subsection, we discuss the experimental results and our system performances.

1) Idiosyncrasy analysis performance: Here the idiosyncratic analysis task was formulated as a 10-class classification problem where the job was to classify the handwritten patches into idiosyncratic classes $\left(\mathrm{ID}_{1}, \mathrm{ID}_{2}, \ldots, \mathrm{ID}_{10}\right)$.

We conducted our experiment of idiosyncratic handwriting analysis separately on the previously mentioned three databases $D_{\mathrm{DAR}}, \mathrm{D}_{\mathrm{CM}}$, and $\mathrm{D}_{\text {new }}$. Each of these databases with subjective idiosyncratic opinion score was divided into training, validation, and test set in 2:1:1 ratio.

The performance of our system for idiosyncratic handwritten text-patch identification is presented in TABLE I Here we show the overall average F-Measure (\%) of all the classes. Since the idiosyncratic text-patch ground-truths are dependent on experts' cognitive analysis, all classes may not be well balanced. Therefore, our database is quite imbalanced. 


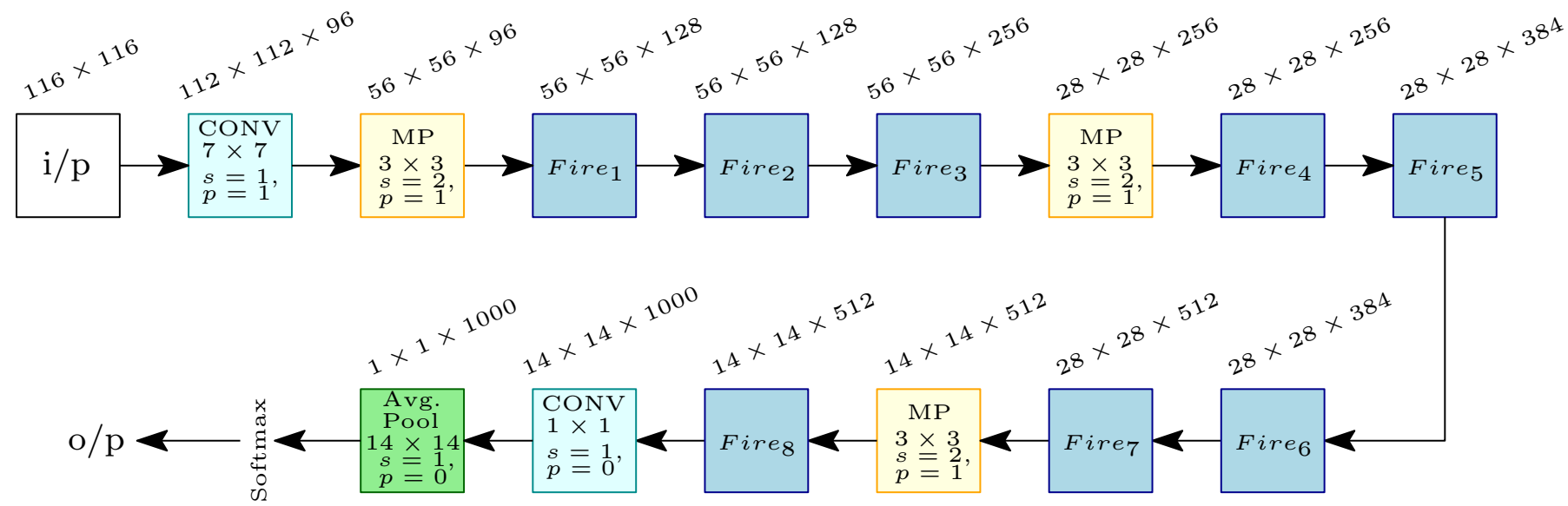

Fig. 7. SqueezeNet architecture.

For classification into such classes, the balanced accuracy provides better performance measure [25]. In TABLE I] we also show the performance of idiosyncrasy analysis in terms of balanced accuracy (\%).

TABLE I

IDIOSYNCRATIC HANDWRITING ANALYSIS

\begin{tabular}{c|c|c}
\hline Database & $\begin{array}{c}\text { F-Measure } \\
(\boldsymbol{\%})\end{array}$ & $\begin{array}{c}\text { Balanced } \\
\text { Accuracy }(\%)\end{array}$ \\
\hline \hline $\mathrm{D}_{\text {DAR }}$ & $\mathbf{9 1 . 3 7}$ & $\mathbf{9 3 . 5 4}$ \\
\hline $\mathrm{D}_{\mathrm{CM}}$ & 89.48 & 91.33 \\
\hline $\mathrm{D}_{\text {new }}$ & 85.96 & 88.16 \\
\hline $\mathrm{D}_{\text {DAR }}+\mathrm{D}_{\mathrm{CM}}+\mathrm{D}_{\text {new }}$ & 89.03 & 90.89 \\
\hline
\end{tabular}

On idiosyncrasy analysis, our system obtained the highest performance on database $\mathrm{D}_{\mathrm{DAR}}$ and lowest performance on $\mathrm{D}_{\text {new }}$. For idiosyncratic handwritten text-patch identification, we obtained $91.37 \%, 89.48 \%, 85.96 \%$ of F-measure and $93.54 \%, 91.33 \%, 88.16 \%$ of balanced accuracy on $\mathrm{D}_{\mathrm{DAR}}$, $\mathrm{D}_{\mathrm{CM}}, \mathrm{D}_{\text {new }}$ databases, respectively. Overall, union of all our employed datasets attained system performance of $89.03 \% \mathrm{~F}$ measure and $90.89 \%$ balanced accuracy.

2) Writer identification performance: In this subsection, we present the experimentations performed for writer identification task to investigate whether there exists any influence of idiosyncratic handwriting on this task.

The databases $D_{\text {DAR }}$ and $D_{C M}$ were not suitable for writer identification task, since writer information was missing. The $D_{\text {new }}$ contained the writer information and was suitable for our study. To inspect the effect of idiosyncratic handwriting on writer identification, all the obtained text-patches (refer to Section (II) of $\mathrm{D}_{\text {new }}$ were subdivided into training, validation, and test set in 2:1:1 ratio.

As previously mentioned in Section III-A and Section III-B1 the handwritten text-patches were classified into 10 idiosyncratic classes $\left(\mathrm{ID}_{1}, \mathrm{ID}_{2}, \ldots, \mathrm{ID}_{10}\right)$. The handwritten samples of class $\mathrm{ID}_{1}$ were highly idiosyncratic whereas $\mathrm{ID}_{10}$ contained least idiosyncratic handwritings. We intended to analyze the writer identification performance using only the
TABLE II

Writer IDENTIFICATION PERformance

\begin{tabular}{c|c|c|c}
\hline \multirow{2}{*}{$\begin{array}{c}\text { Idiosyncratic } \\
\text { class (ID) }\end{array}$} & \multicolumn{3}{|c}{ Accuracy (\%) } \\
\cline { 2 - 4 } & Top-1 & Top-2 & Top-5 \\
\hline \hline $\mathrm{ID}_{1}$ & 93.23 & 93.72 & 96.57 \\
\hline $\mathrm{ID}_{2}$ & 92.85 & 93.46 & 95.81 \\
\hline $\mathrm{ID}_{3}$ & 91.66 & 92.18 & 95.10 \\
\hline $\mathrm{ID}_{4}$ & 89.94 & 90.43 & 93.76 \\
\hline $\mathrm{ID}_{5}$ & 87.38 & 87.89 & 91.27 \\
\hline $\mathrm{ID}_{1,2}$ & 94.03 & 94.76 & 97.54 \\
\hline $\mathrm{ID}_{1,3}$ & 93.62 & 93.90 & 96.79 \\
\hline $\mathrm{ID}_{2,3}$ & 92.98 & 93.54 & 95.42 \\
\hline $\mathrm{ID}_{1-3}$ & $\mathbf{9 4 . 4 6}$ & $\mathbf{9 4 . 9 2}$ & $\mathbf{9 8 . 2 7}$ \\
\hline $\mathrm{ID}_{1-4}$ & 93.71 & 94.48 & 97.68 \\
\hline $\mathrm{ID}_{1-5}$ & 93.28 & 94.15 & 96.92 \\
\hline $\mathrm{ID}_{1-10}$ & 90.43 & 91.59 & 94.26 \\
\hline \multicolumn{4}{|c}{}
\end{tabular}

highest idiosyncratic handwriting (class $\mathrm{ID}_{1}$ ) of our database $\mathrm{D}_{\text {new }}$. Besides, we performed the writer identification experiments on different idiosyncratic classes. We also experimented with all possible unary, binary, ternary, quaternary, $\ldots, n$ ary combinations of idiosyncratic classes $\left(\operatorname{ID}_{n \mid n}=1,2, \ldots, 10\right)$. Here, in TABLE II] we present the major results of our writer identification method on some important idiosyncratic classes.

For writer identification performance analysis, we employed the Top- $N$ criterion where a questioned document's writer belonged to a reduced set of ' $\mathrm{N}$ ' ( $\ll$ total number of writers). Here, we selected Top-1,Top-2 and Top-5 criteria for providing the writer identification performance in terms of accuracy (\%). In $D_{\text {new }}$ database, the samples of multiple writer classes were quite balanced.

In TABLE II, ID ID, $_{1,2}$ denotes union of text-patches of the idiosyncratic class- $1\left(\mathrm{ID}_{1}\right)$ and class-2 $\left(\mathrm{ID}_{2}\right)$, i.e., $\mathrm{ID}_{1,2} \equiv$ $\mathrm{ID}_{1} \cup \mathrm{ID}_{2}$. Likewise, $\mathrm{ID}_{1,3} \equiv \mathrm{ID}_{1} \cup \mathrm{ID}_{3}, \mathrm{ID}_{1-3} \equiv \mathrm{ID}_{1} \cup \mathrm{ID}_{2} \cup \mathrm{ID}_{3}$, $\mathrm{ID}_{1,2,4} \equiv \mathrm{ID}_{1} \cup \mathrm{ID}_{2} \cup \mathrm{ID}_{4}$ etc., and $\mathrm{ID}_{1-10}$ denotes the union of text-patches of all idiosyncratic classes. As a matter of fact, experimenting on $\mathrm{ID}_{1-10}$ is synonymous to the traditional writer identification experiment on the full page of writing.

With reference to TABLE II] we obtained $93.23 \%$ Top1 writer identification accuracy while employing only the 
highest idiosyncratic handwriting (class $\mathrm{ID}_{1}$ ). On this $\mathrm{ID}_{1}$, the attained Top-2 and Top-5 accuracies were $93.72 \%$ and $96.57 \%$, respectively.

Among binary combinations (e.g. $\mathrm{ID}_{1,2}, \mathrm{ID}_{1,3}, \mathrm{ID}_{2,3}$ etc.) of the idiosyncratic classes, highest Top-1 accuracy (94.03\%) was obtained on $\mathrm{ID}_{1,2}$. Among ternary combinations (e.g. ID $_{1-3}$, $\mathrm{ID}_{2-4}, \mathrm{ID}_{1,2,4}$ etc.), highest $94.46 \%$ of Top- 1 accuracy was obtained on $\mathrm{ID}_{1-3}$. It was the best Top- 1 accuracy among all possible $n$-ary combinations of idiosyncratic classes.

Union of all the idiosyncratic classes (ID ${ }_{1-10}$ ) produced $90.43 \%$ of Top- 1 accuracy. In other words, classical experimentation on all the text-patches attained $90.43 \%$ Top- 1 writer identification accuracy. However, each of the upper three idiosyncratic classes $\left(\mathrm{ID}_{1}, \mathrm{ID}_{2}\right.$, and $\left.\mathrm{ID}_{3}\right)$ performed better than the traditional approach of considering all text components. Thus, from our experimental results, it can be noted that highly idiosyncratic handwriting can improve the writer identification performance. Here, experimenting on $\mathrm{ID}_{1}$ and $\mathrm{ID}_{1-3}$, we obtained respective $2.8 \%$ and $4.03 \%$ additional Top- 1 writer identification accuracy when compared to $\mathrm{ID}_{1-10}$. This baseline performance added value to our idiosyncratic analysis.

\section{Comparison}

To the best of our knowledge, this work is the earliest attempt of its kind on idiosyncratic handwriting analysis. We did not find any related work for making a comparison.

However, it was interesting to see that highly idiosyncratic handwritings produced up to $4.03 \%$ additional Top- 1 writer identification accuracy on our experimentations when compared to the same writer identification method without using idiosyncratic analysis (refer to Section III-B2).

\section{CONCLUSION}

In this paper, we scrutinize the idiosyncrasy of individual handwriting and show its facilitative nature for the writer identification task. The idiosyncratic handwriting analysis task is modeled as a machine learning problem with the aid of cognitive score of handwriting experts. Here, an Inception network is employed for idiosyncratic text inspection and the SqueezeNet is used for writer identification. For both of these tasks, auto-derived deep features are used. Experimentation of idiosyncratic handwriting analysis is performed on two publicly available and one in-house offline Bengali handwritten databases with collected subjective opinion ground-truth. Overall $90.89 \%$ balanced accuracy has been obtained for the idiosyncrasy analysis task on these three datasets. The writer identification job has experimented on the in-house database. For unary idiosyncratic classes, we have obtained highest 93.23\% Top- 1 writer identification accuracy (on $\mathrm{ID}_{1}$ ). The best Top- 1 accuracy of $94.46 \%$ has been obtained while combining three highly unary idiosyncratic classes $\left(\mathrm{ID}_{1}, \mathrm{ID}_{2}\right.$, and $\left.\mathrm{ID}_{3}\right)$. Our experiments suggest that prior analyzing of idiosyncratic handwriting can improve the writer identification performance up to $4.03 \%$ (Top-1 accuracy).
Although in this paper, we have worked on Bengali handwriting, our method can be extended to some other scripts. In future, we will try to exploit the idiosyncrasy analysis for multilingual handwriting. We also plan to hybridize different neural networks for a comparative study.

\section{ACKNOWLEDGMENT}

We thank all volunteers and handwriting experts for their help in database generation.

\section{REFERENCES}

[1] M. Bulacu, L. Schomaker, "Text-Independent Writer Identification and Verification Using Textural and Allographic Features", IEEE Trans. on PAMI, vol. 29, no. 4, pp. 701-717, 2007.

[2] R.D. Banerji, "The Origin of the Bengali Script", University of Calcutta, 1919.

[3] B.B. Chaudhuri, U. Pal, "A Complete Printed Bangla OCR System", Pattern Recognition, vol. 31, no. 5, pp. 531-549, 1998.

[4] J.J. Schneider, "Contexts, Genres, and Imagination: An Examination of the Idiosyncratic Writing Performances of Three Elementary Children within Multiple Contexts of Writing Instruction", Research in the Teaching of English, vol. 37, no. 3, pp. 329-379, 2003.

[5] T.S.F. Haines, O.M. Aodha, G.J. Brostow, "My Text in Your Handwriting", ACM Trans. on Graphics, vol. 35, no. 3, artl. 26, 18 pages, 2016.

[6] J. Chen, W. Cheng, D.P. Lopresti, "Using Perturbed Handwriting to Support Writer Identification in the Presence of Severe Data Constraints", Proc. DRR XVIII, vol. 7874, 78740G, 2011.

[7] C. Adak, B.B. Chaudhuri, M. Blumenstein, "Offline Cursive Bengali Word Recognition using CNNs with a Recurrent Model”, Proc. ICFHR, pp. 429-434, 2016.

[8] C. Szegedy et al., "Going Deeper with Convolutions", Proc. IEEE Conf. on Computer Vision and Pattern Recognition (CVPR), pp. 1-9, 2015.

[9] R.A. Huber, A.M. Headrick, "Handwriting Identification: Facts and Fundamentals", CRC Press, 1999.

[10] U. Porwal et al., "Multiclass Learning for Writer Identification Using Error-Correcting Codes", Proc. DAS, pp. 16-20, 2014.

[11] F.N. Iandola et al., "SqueezeNet: AlexNet-level Accuracy with 50x Fewer Parameters and <0.5MB Model Size", CoRR abs/1602.07360.

[12] F. Zhao, H.Z. Lu, Z.Y. Zhang, "Real-time Single-pass Connected Components Analysis Algorithm", EURASIP Journal on Image and Video Processing, vol. 21, pp. 1-10, 2013.

[13] C. Adak, B.B. Chaudhuri, "Extraction of Doodles and Drawings from Manuscripts", Proc. Int. Conf. on Pattern Recognition and Machine Intelligence (PReMI), LNCS \#8251, pp. 515-520, 2013.

[14] B.B. Chaudhuri, C. Adak, "An Approach for Detecting and Cleaning of Struck-out Handwritten Text”, Pattern Recognition, vol. 61, pp. 282-294, 2017.

[15] U. Pal, S. Datta, "Segmentation of Bangla Unconstrained Handwritten Text", Proc. ICDAR, pp. 1128-1132, 2003.

[16] R. Likert, "A Technique for the Measurement of Attitudes", Archives of Psychology, New York, no. 140, pp. 1-55, 1932.

[17] Q.H.-Thu, M.N. Garcia, F. Speranza, P. Corriveau, A. Raake, "Study of Rating Scales for Subjective Quality Assessment of High-Definition Video", IEEE Trans. on Broadcasting, vol. 57, no. 1, pp. 1-14, 2011.

[18] Y. LeCun et al., "Gradient-based Learning Applied to Document Recognition", Proceedings of the IEEE, vol. 86, no. 11, pp. 2278-2324, 1998

[19] V. Nair, G.E. Hinton, "Rectified Linear Units Improve Restricted Boltzmann Machines", Proc. ICML, pp. 807-814, 2010

[20] M. Lin et al., "Network in Network", CoRR, abs/1312.4400, 2013.

[21] N. Srivastava, G.E. Hinton, A. Krizhevsky, I. Sutskever, R. Salakhutdinov, "Dropout: A Simple Way to Prevent Neural Networks from Overfitting", JMLR, vol. 15, no. 1, pp. 1929-1958, 2014.

[22] A. Krizhevsky et al., "ImageNet Classification with Deep Convolutional Neural Networks", Proc. NIPS, vol. 1, pp. 1097-1105, 2012.

[23] N. Stamatopoulos et al., "ICDAR 2013 Handwriting Segmentation Contest", Proc. ICDAR, pp. 1402-1406, 2013.

[24] R. Sarkar et al., "CMATERdb1: A Database of Unconstrained Handwritten Bangla and Bangla-English Mixed Script Document Image", IJDAR, vol. 15 , no. 1 , pp.71-83, 2012

[25] K.H. Brodersen et al., "The Balanced Accuracy and Its Posterior Distribution", Proc. ICPR, pp. 3121-3124, 2010. 